the time of heart transplantation after VAD support (post-VAD group). Normalized levels of iNOS expression were compared by extracting protein from tissue samples followed by western blotting analysis. Levels of cardioymyocyte apoptosis were analyzed using terminal deoxynucleotidyltransferase deoxyuridine triphosphate nick end-labeling (TUNEL) staining on pre-VAD and post-VAD myocardial tissue samples.

Higher levels of iNOS protein were detected in transplant and pre-VAD myocardial samples compared to controls, consistent with previous reports. Interestingly, myocardial iNOS protein levels were reported to normalize after VAD therapy. TUNEL assay revealed higher levels of cardiomyocyte apoptosis in the pre-VAD group compared to the post-VAD group, and levels of iNOS protein correlated significantly with the percentage of TUNEL-positive cardiomyocytes.

Patten et al. conclude that VAD therapy normalizes iNOS expression in association with reduced cardiomyocyte apoptosis, suggesting a causal link between iNOS and cardiomyocyte apoptosis in the failing human heart.

Original article Patten RD et al. (2005) Ventricular assist device therapy normalizes inducible nitric oxide synthase expression and reduces cardiomyocyte apoptosis in the failing human heart. J Am Coll Cardiol 45: 1419-1424

\section{Measuring 'aspirin resistance': point-of-care platelet- function tests versus optical aggregometry}

Aspirin is widely prescribed for the secondary prevention of ischemic stroke and acute coronary syndromes, but some patients do not respond to this therapy. There is evidence that these 'aspirin-resistant' patients are at an increased risk of ischemic vascular events, and so point-of-care platelet-function assays have been introduced. Simpler to use than light-transmission aggregometry (LTA), these tests are designed to allow the routine clinical assessment of aspirin responsiveness. Whether the tests agree with LTA is the subject of a recent study published in Stroke.

Harrison and colleagues recruited 100 patients with transient ischemic attack or stroke, from the ongoing Oxford Vascular Study. All had been taking $75-150 \mathrm{mg}$ aspirin daily for at least 4 weeks prior to the study. Blood samples were subjected to platelet-function assay using two point-of-care systems: the PFA-100 test (Dade-Behring Inc., Deerfield, IL) and the Ultegra RPFA test (Accumetrics Inc., San Diego, CA). Results were compared with those obtained by LTA, which was performed using a Biodata-PAP-4 aggregometer (Alpha Laboratories Ltd., Eastleigh, Hants, UK).

The analysis showed that aspirin nonresponsiveness is highly test-specific, with an incidence of $22 \%$ when measured using the PFA-100, $17 \%$ using the RPFA, and only $5 \%$ using LTA. Only $2 \%$ of patients were nonresponders by all three tests. Harrison et al. conclude, therefore, that large, prospective studies are needed to establish the prognostic value of each of these methods.

Original article Harrison P et al. (2005) Screening for aspirin responsiveness after transient ischemic attack and stroke: comparison of 2 point-of-care platelet function tests with optical aggregometry. Stroke 36: 1001-1005

\section{The Euro Heart Survey on infective endocarditis}

Results from the Euro Heart Survey on valvular heart disease-spanning 92 centers in 25 countries-have revealed that active infective endocarditis (IE) is still associated with a high mortality rate, and that improvements could be made in the implementation of guidelines and patient education.

Of 5,001 adult patients with valvular heart disease included in the survey, $159(3 \%)$ had active IE and were included in the recent analysis. Of these patients, 118 (74\%) had native IE and $41(26 \%)$ had prosthetic IE. Most patients presented with fever and heart failure was common. In-hospital mortality was $12.6 \%$.

Although the vast majority of patients received intravenous antibiotics for at least 1 month, blood cultures were taken in only $71 \%$ of cases; a surprisingly high proportion of these (14\%) were negative. Approximately half of the patients underwent surgery for at least one indication, including heart failure $(60 \%)$, persistent sepsis $(40 \%)$, vegetation size (48\%), or embolism (18\%). Implantation of a mechanical prosthesis was the most common type of surgery.

Among the total survey population, only half of the patients with native valvular heart disease had received information on endocarditis prophylaxis, and only a third had 